\title{
An Efficient Method for Solving System of Third-Order Nonlinear Boundary Value Problems
}

\author{
Muhammad Noor, ${ }^{1,2}$ Khalida Noor, $^{1}$ Asif Waheed, ${ }^{1}$ \\ and Eisa A. Al-Said ${ }^{2}$ \\ ${ }^{1}$ Department of Mathematics, COMSATS Institute of Information Technology, Park Road, \\ Chak Shahzad, Islamabad, Pakistan \\ ${ }^{2}$ Mathematics Department, College of Science, King Saud University, Riyadh, Saudi Arabia \\ Correspondence should be addressed to Muhammad Noor, noormaslam@hotmail.com
}

Received 24 November 2010; Accepted 13 April 2011

Academic Editor: P. Liatsis

Copyright (C) 2011 Muhammad Noor et al. This is an open access article distributed under the Creative Commons Attribution License, which permits unrestricted use, distribution, and reproduction in any medium, provided the original work is properly cited.

we use the modified variation of parameters method for finding the analytical solution of a system of third-order nonlinear boundary value problems associated with obstacle, unilateral, and contact problems. The results are calculated in terms of convergent series with easily computable components. The suggested technique is applied without any discretization, perturbation, transformation, and restrictive assumptions. Moreover, it is free from round off errors. Some examples are given to illustrate the implementation and efficiency of the modified variation of parameters method.

\section{Introduction}

In recent years, much attention has been given to solve system of third-order boundary value problems, see [1-11]. In this paper, we consider the following systems of third-order nonlinear boundary value problems:

$$
\begin{gathered}
u^{\prime \prime \prime}= \begin{cases}f(x, u(x)), & a \leq x<c, \\
f(x, u(x))+u(x) g(x)+r, & c \leq x<d, \\
f(x, u(x)), & d \leq x \leq b,\end{cases} \\
u^{\prime \prime \prime}= \begin{cases}f(x, u(x))+u(x) g(x)+r, & a \leq x<c, \\
f(x, u(x)), & c \leq x<d, \\
f(x, u(x))+u(x) g(x)+r, & d \leq x \leq b,\end{cases}
\end{gathered}
$$


with boundary conditions $u(a)=\alpha_{1}, u^{\prime}(a)=\alpha_{2}, u(b)=\alpha_{3}$, and continuity conditions of $u(x), u^{\prime}(x)$, and $u^{\prime \prime}(x)$ at internal points $c$ and $d$ of the interval $[a, b]$. Here $r$ and $\alpha_{i}, i=1, \ldots, 3$ are real and finite constants, $g(x)$ is a continuous function on $[a, b]$ and $f(x, u(x))=f(u)$ is a nonlinear function. Such type of problems arise in the study of obstacle, contact, unilateral, and equilibrium problems arising in economics, transportation, nonlinear optimization, oceanography, ocean wave engineering, fluid flow through porous media, and some other branches of pure and applied sciences; see [1, 2, 4-15] and references therein. Several techniques have been used to solve system of linear third-order boundary value problems associated with obstacle, contact, and unilateral problems. Noor et al. [7] applied finite difference method for unilateral problems; Al-Said et al. [14] used finite difference method for obstacle problems; Khalifa and Noor [15] applied quintic spline method for contact problems; Noor and Khalifa [8] applied quartic spline method for odd-order obstacle problems; Al-Said and Noor [13] used quartic spline method for obstacle problems. Gao and Chi [1] applied quartic B-spline method for third-order obstacle problems and Islam et al. [11] proposed nonpolynomial spline methods for solving system of third-order boundary value problems associated with obstacle, contact, and unilateral problems. Some of these methods are numerical and require huge computational work for finding the approximate solutions. Moreover, these numerical methods provide discrete point solution.

In this paper, we use the modified variation of parameters method to solve the systems of third-order nonlinear boundary value problems associated with obstacle problems. Noor et al. $[4,5,10,16,17]$ have used variation of parameters method for solving a wide classes of higher-order initial and boundary value problems. Ma et al. [18-20] have applied variation of parameters method for solving some nonhomogenous partial differential equations. Ramos [21] has used this technique to find frequency of some nonlinear oscillators. Ramos [21] has also shown the equivalence of this technique with variational iteration method. It is further investigated by Noor et al. $[4,5,10,16,17]$ that the proposed technique is distinctly different from variational iteration method in many aspects. The multiplier used in the variation of parameters method is obtained by Wronskian technique and is totally different from the Lagrange multiplier of variational iterative method. Moreover, the variation of parameters method (VPM) removes the higher-order derivative term from its iterative scheme which is clear advantage over the variational iteration method (VIM) as the term may cause of repeated computation and computations of unneeded terms, which consumes time and effort, in most of the cases, one of the cases is mentioned in [22]. Thus the variation of parameters method has reduced a lot of computational work involved due to this term as compared to some other existing techniques using this term. This shows that the variation of parameters method is better than the other techniques. The modified variation of parameters method is obtained by combining the variation of parameters method $[3-5,19,20]$ and Adomian's decomposition method [23]. It turned out that modified variation of parameters method is very flexible and is very efficient. The use of multiplier and Adomian's polynomial together in the modified variation of parameters method increases the rate of convergence by reducing the number of iterations and successive application of integral operators. This technique makes the solution procedure simple while still maintaining the higher level of accuracy. Here, we implement this technique for solving systems of third-order nonlinear boundary value problems associated with obstacle, unilateral, and contact problems, which is the main motivation of this paper. Examples are given to illustrate the implementation and the efficiency of the proposed method. The comparison of the proposed method with other similar methods is the subject of another paper. It is well known that the obstacle problems can be studied in the general frame work of the variational inequalities. The applications 
of the modified variation of parameters methods for solving the variational inequalities is an open and interesting problem for future research. For the formulation, applications, and numerical techniques for solving the variational inequalities and related optimization problems, see $[2-4,7,24,25]$ and the references therein.

\section{Modified Variation of Parameters Method}

To illustrate the basic concept of the variation of parameter method for differential equations, we consider the general differential equation in operator form

$$
L u(x)+R u(x)+N u(x)=g(x)
$$

where $L$ is a higher-order linear operator, $R$ is a linear operator of order less than $L, N$ is a nonlinear operator, and $g$ is a source term. sing variation of parameters method $[2-5,10,16$, 17], we have following general solution of (2.1)

$$
u(x)=\sum_{i=0}^{n-1} \frac{B_{i} x^{i}}{i !}+\int_{0}^{x} \lambda(x, s)(-N u(s)-R u(s)+g(s)) d s,
$$

where $n$ is a order of given differential equation and $B i^{\prime} s$ are unknowns which can be further determined by initial/boundary conditions. Here $\lambda(x, s)$ is multiplier which can be obtained with the help of Wronskian technique. This multiplier removes the successive application of integrals in iterative scheme, and it depends upon the order of equation. Noor et al. $[4,5,16$, 17] have obtained the following for finding the multiplier $\lambda(x, s)$ as

$$
\lambda(x, s)=\sum_{i=1}^{n} \frac{s^{i-1} x^{n-i}(-1)^{i-1}}{(i-1) !(n-i) !} .
$$

For different choices of $n$, one can obtain the following values of $\lambda$

$$
\begin{aligned}
& n=1, \quad \lambda(x, s)=1, \\
& n=2, \quad \lambda(x, s)=x-s, \\
& n=3, \quad \lambda(x, s)=\frac{x^{2}}{2 !}-s x+\frac{s^{2}}{2 !}, \\
& n=4, \quad \lambda(x, s)=\frac{x^{3}}{3 !}-\frac{s x^{2}}{2 !}+\frac{s^{2} x}{2 !}-\frac{s^{3}}{3 !},
\end{aligned}
$$

Hence, we have the following iterative scheme from (2.2):

$$
u_{k+1}(x)=u_{k}(x)+\int_{0}^{x} \lambda(x, s)\left(-N u_{k}(s)-R u_{k}(s)+g(s)\right) d s, \quad k=0,1,2, \ldots
$$

It is observed that the fix value of initial guess in each iteration provides the better approximation, that is, $u_{k}(x)=u_{0}(x)$, for $k=1,2, \ldots$ However, we can modify the initial guess by 
dividing $u_{0}(x)$ in two parts and using one of them as initial guess. It is more convenient way in case of more than two terms in $u_{0}(x)$. In a modified variation of parameters method, we define the solution $u(x)$ by the following series:

$$
u(x)=\sum_{k=0}^{\infty} u_{k}(x)
$$

and the nonlinear terms are decomposed by infinite number of polynomials as follows:

$$
N(u)=\sum_{k=0}^{\infty} A_{k}\left(u_{0}, u_{1}, u_{2}, \ldots, u_{i}\right)
$$

where $u$ is a function of $x$ and $A_{k}$ are the so-called Adomian's polynomials. These polynomials can be generated for various classes of nonlinearities by specific algorithm developed in [23] as follows:

$$
A_{k}=\left(\frac{1}{k !}\right)\left(\frac{d^{k}}{d \lambda^{k}}\right) N\left(\sum_{i=0}^{n}\left(\lambda^{i} u_{i}\right)\right)_{\lambda=0}, \quad k=0,1,2, \ldots
$$

Hence, we have the following iterative scheme for finding the approximate solution of (2.1) as

$$
u_{k+1}(x)=u_{k}(x)+\int_{0}^{x} \lambda(x, s)\left(-A_{k}-R u_{k}(s)+g(s)\right) d s
$$

We would like to mention that the modified variation of parameters method for solving the system of third-order nonlinear boundary value problems may be viewed as an important and significant improvement as compared with other similar method.

\section{Numerical Results}

Example 3.1. Consider following system of third-order nonlinear boundary value problems relevant to system (1.1):

$$
u^{\prime \prime \prime}=\left\{\begin{array}{lc}
\frac{u^{3}}{3 !}+\frac{u^{2}}{2 !}+u+1, & \text { for }-1 \leq x<-\frac{1}{2} \\
\frac{u^{3}}{3 !}+\frac{u^{2}}{2 !}-3 u+2, & \text { for }-\frac{1}{2} \leq x<\frac{1}{2} \\
\frac{u^{3}}{3 !}+\frac{u^{2}}{2 !}+u+1, & \text { for } \frac{1}{2} \leq x \leq 1,
\end{array}\right.
$$

with boundary conditions $u(-1)=u(1)=0, u^{\prime}(-1)=1$. 
We will use modified variation of parameters method for solving system of third-order nonlinear boundary value problems (3.1). By using the modified variation of parameters method, we have following iterative scheme to solve nonlinear system (3.1):

$$
u_{k+1}(x)= \begin{cases}u_{k}(x)+\int_{0}^{x} \lambda(x, s)\left(A_{k}+u_{k}+1\right) d s, & \text { for }-1 \leq x<-\frac{1}{2} \\ u_{k}(x)+\int_{0}^{x} \lambda(x, s)\left(A_{k}-3 u_{k}+2\right) d s, & \text { for }-\frac{1}{2} \leq x<\frac{1}{2} \\ u_{k}(x)+\int_{0}^{x} \lambda(x, s)\left(A_{k}+u_{k}+1\right) d s, & \text { for } \frac{1}{2} \leq x \leq 1 .\end{cases}
$$

Since the governing equation is of 3 rd order, using $\lambda(x, s)=x^{2} / 2 !-x s-s^{2} / 2$ !, we have

$$
u_{k+1}(x)= \begin{cases}u_{k}(x)+\int_{0}^{x}\left(\frac{x^{2}}{2 !}-x s-\frac{s^{2}}{2 !}\right)\left(A_{k}+u_{k}+1\right) d s, & \text { for }-1 \leq x<-\frac{1}{2} \\ u_{k}(x)+\int_{0}^{x}\left(\frac{x^{2}}{2 !}-x s-\frac{s^{2}}{2 !}\right)\left(A_{k}+2 u_{k}\right) d s, & \text { for }-\frac{1}{2} \leq x<\frac{1}{2} \\ u_{k}(x)+\int_{0}^{x}\left(\frac{x^{2}}{2 !}-x s-\frac{s^{2}}{2 !}\right)\left(A_{k}+u_{k}+1\right) d s, & \text { for } \frac{1}{2} \leq x \leq 1\end{cases}
$$

Case $1(-1 \leq x<-1 / 2)$. In this case, we implement the modified variation of parameters method as follows: we take $u_{0}=c_{1}\left(x^{2} / 2\right.$ !) $+c_{2} x+c_{3}$, for better approximation. we decompose initial guess as $u_{0}=c_{2} x$, and obtain further iterations as follows:

$$
\begin{aligned}
u_{1}(x)= & c_{3}+c_{1} \frac{x^{2}}{2 !}+\int_{0}^{x}\left(\frac{x^{2}}{2 !}-s x+\frac{s^{2}}{2 !}\right)\left(A_{0}+u_{0}+1\right) d s \\
u_{1}(x)= & c_{3}+\frac{1}{2} c_{1} x^{2}+\frac{1}{6} x^{3}+\frac{1}{24} c_{2} x^{4}+\frac{1}{120} c_{2}{ }^{2} x^{5}+\frac{1}{720} c_{2}{ }^{3} x^{6} \\
u_{k+2}(x)= & \int_{0}^{x}\left(\frac{x^{2}}{2 !}-s x+\frac{s^{2}}{2 !}\right)\left(A_{k+1}+u_{k+1}\right) d s, \quad \text { for } k=0,1,2, \ldots, \\
u_{2}(x)= & \frac{1}{6} c_{3} x^{3}+\frac{1}{24} c_{2} c_{3} x^{4}+\left(\frac{1}{120} c_{1}+\frac{1}{24} c_{2} c_{3}\right) x^{5}+\left(\frac{1}{720}+\frac{1}{240} c_{1} c_{2}\right) x^{6} \\
& +\left(\frac{1}{1008}+\frac{1}{840} c_{1} c_{2}\right) c_{2} x^{7}+\frac{1}{2520} c_{2}{ }^{2} x^{8}+\frac{11}{181440} c_{2}{ }^{3} x^{9}+\frac{1}{129600} c_{2}{ }^{4} x^{10} \\
& +\frac{11}{1425600} c_{2}{ }^{5} x^{11}
\end{aligned}
$$


Case $2(-1 / 2 \leq x<1 / 2)$. In this case, we have following approximations:

$$
\begin{aligned}
u_{0}= & c_{5} x \\
u_{1}(x)= & c_{6}+c_{4} \frac{x^{2}}{2 !}+\int_{0}^{x}\left(\frac{x^{2}}{2 !}-s x+\frac{s^{2}}{2 !}\right)\left(A_{0}+2 u_{0}\right) d s \\
u_{1}(x)= & c_{6}+\frac{1}{2} c_{4} x^{2}+\frac{1}{12} c_{5} x^{4}+\frac{1}{120} c_{5}{ }^{2} x^{5}+\frac{1}{720} c_{5}{ }^{3} x^{6} \\
u_{k+2}(x)= & \int_{0}^{x}\left(\frac{x^{2}}{2 !}-s x+\frac{s^{2}}{2 !}\right)\left(A_{k+1}+2 u_{k+1}\right) d s, \text { for } k=0,1,2, \ldots \\
u_{2}(x)= & \frac{1}{3} c_{6} x^{3}+\frac{1}{24} c_{5} c_{6} x^{4}+\left(\frac{1}{60} c_{4}+\frac{1}{120} c_{5}{ }^{2} c_{6}\right) x^{5}+\frac{1}{240} c_{4} c_{5} x^{6} \\
& +\left(\frac{1}{1260} c_{5}+\frac{1}{840} c_{5}{ }^{2} c_{4}\right) c_{2} x^{7} \\
& +\frac{1}{3360} c_{5}{ }^{2} x^{8}+\frac{19}{181440} c_{5}{ }^{3} x^{9}+\frac{1}{129600} c_{5}{ }^{4} x^{10}+\frac{11}{1425600} c_{5}{ }^{5} x^{11},
\end{aligned}
$$

Case $3(1 / 2 \leq x \leq 1)$. In this case, we proceed as follows:

$$
\begin{aligned}
u_{0}= & c_{8} x \\
u_{1}(x)= & c_{9}+c_{7} \frac{x^{2}}{2 !}+\int_{0}^{x}\left(\frac{x^{2}}{2 !}-s x+\frac{s^{2}}{2 !}\right)\left(A_{0}+u_{0}+1\right) d s \\
u_{1}(x)= & c_{9}+\frac{1}{2} c_{7} x^{2}+\frac{1}{6} x^{3}+\frac{1}{24} c_{8} x^{4}+\frac{1}{120} c_{8}{ }^{2} x^{5}+\frac{1}{720} c_{8}{ }^{3} x^{6} \\
u_{k+2}(x)= & \int_{0}^{x}\left(\frac{x^{2}}{2 !}-s x+\frac{s^{2}}{2 !}\right)\left(A_{k+1}+u_{k+1}\right) d s, \quad \text { for } k=0,1,2, \ldots, \\
u_{2}(x)= & \frac{1}{6} c_{9} x^{3}+\frac{1}{24} c_{8} c_{9} x^{4}+\left(\frac{1}{120} c_{7}+\frac{1}{24} c_{8} c_{9}\right) x^{5}+\left(\frac{1}{720}+\frac{1}{240} c_{7} c_{8}\right) x^{6} \\
& +\left(\frac{1}{1008}+\frac{1}{840} c_{7} c_{8}\right) c_{2} x^{7}+\frac{1}{2520} c_{8}{ }^{2} x^{8} \\
& +\frac{11}{181440} c_{8}{ }^{3} x^{9}+\frac{1}{129600} c_{8}{ }^{4} x^{10}+\frac{11}{1425600} c_{8}{ }^{5} x^{11}
\end{aligned}
$$


By using MVPM, we have following formula for getting series solution in the whole domain from the above cases:

$$
u(x)= \begin{cases}\sum_{k=0}^{\infty} u_{k}(x), & \text { for }-1 \leq x \leq-\frac{1}{2} \\ \sum_{k=0}^{\infty} u_{k}(x), & \text { for }-\frac{1}{2} \leq x \leq \frac{1}{2} \\ \sum_{k=0}^{\infty} u_{k}(x), & \text { for } \frac{1}{2} \leq x \leq 1\end{cases}
$$

Hence, we have the following series solution after two iterations:

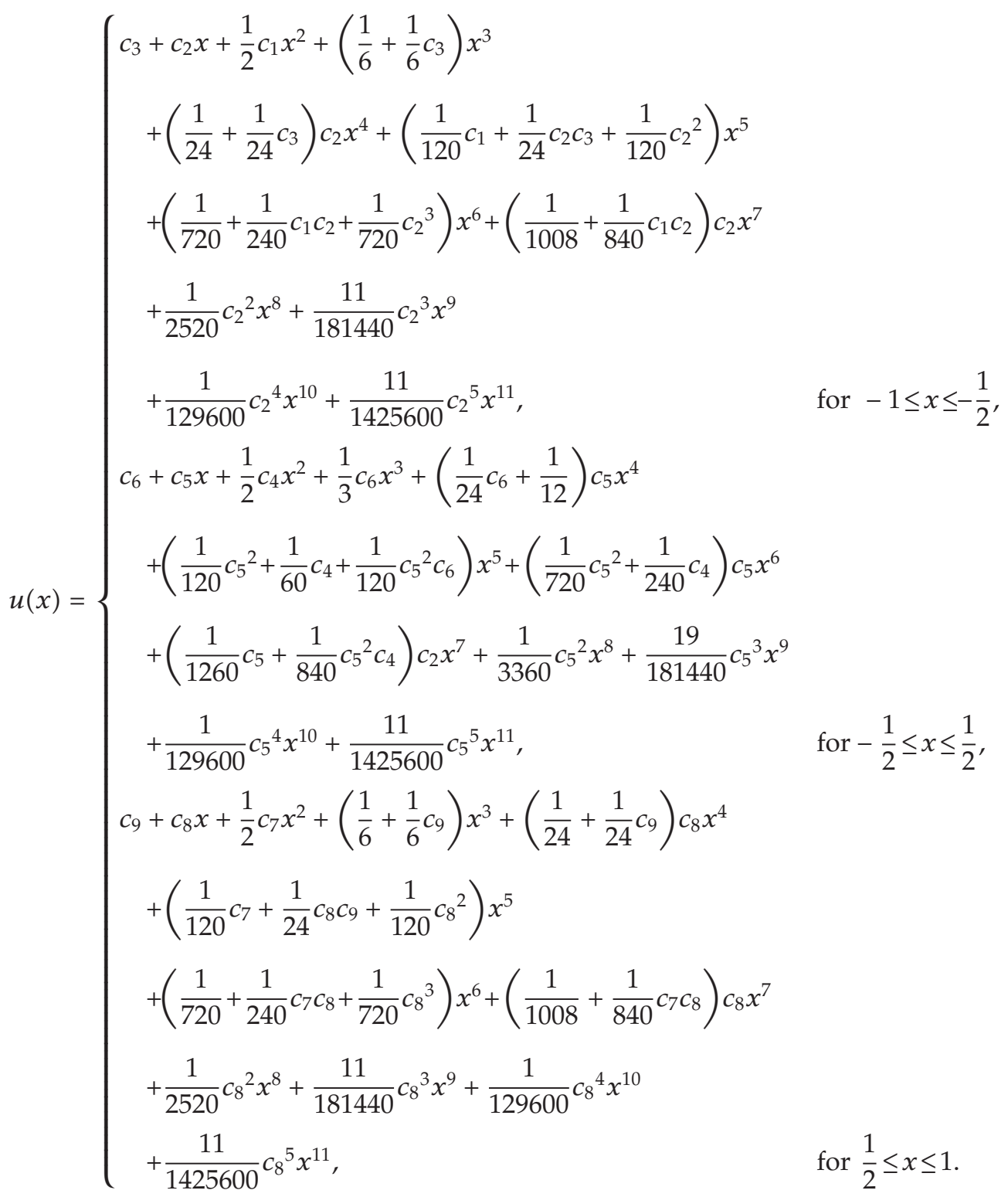


By using boundary conditions and continuity conditions at $x=-1 / 2$ and $x=1 / 2$ and we have a system of nonlinear equations. By using Newton's method for system of nonlinear equations, we have the following values of unknown constants:

$$
\begin{array}{lll}
c_{1}=1.6049253290, & c_{2}=.2878849923, & c_{3}=-.4129294970, \\
c_{4}=.8760312015, & c_{5}=.1067238767, & c_{6}=-.4430093667, \\
c_{7}=.1999018898, & c_{8}=.2719677907, & c_{9}=-.4701798231 .
\end{array}
$$

By using values of unknowns from (3.11) into (3.10), we have following analytic solution of system of forth-order nonlinear boundary value problem associated with obstacle problem(3.1)

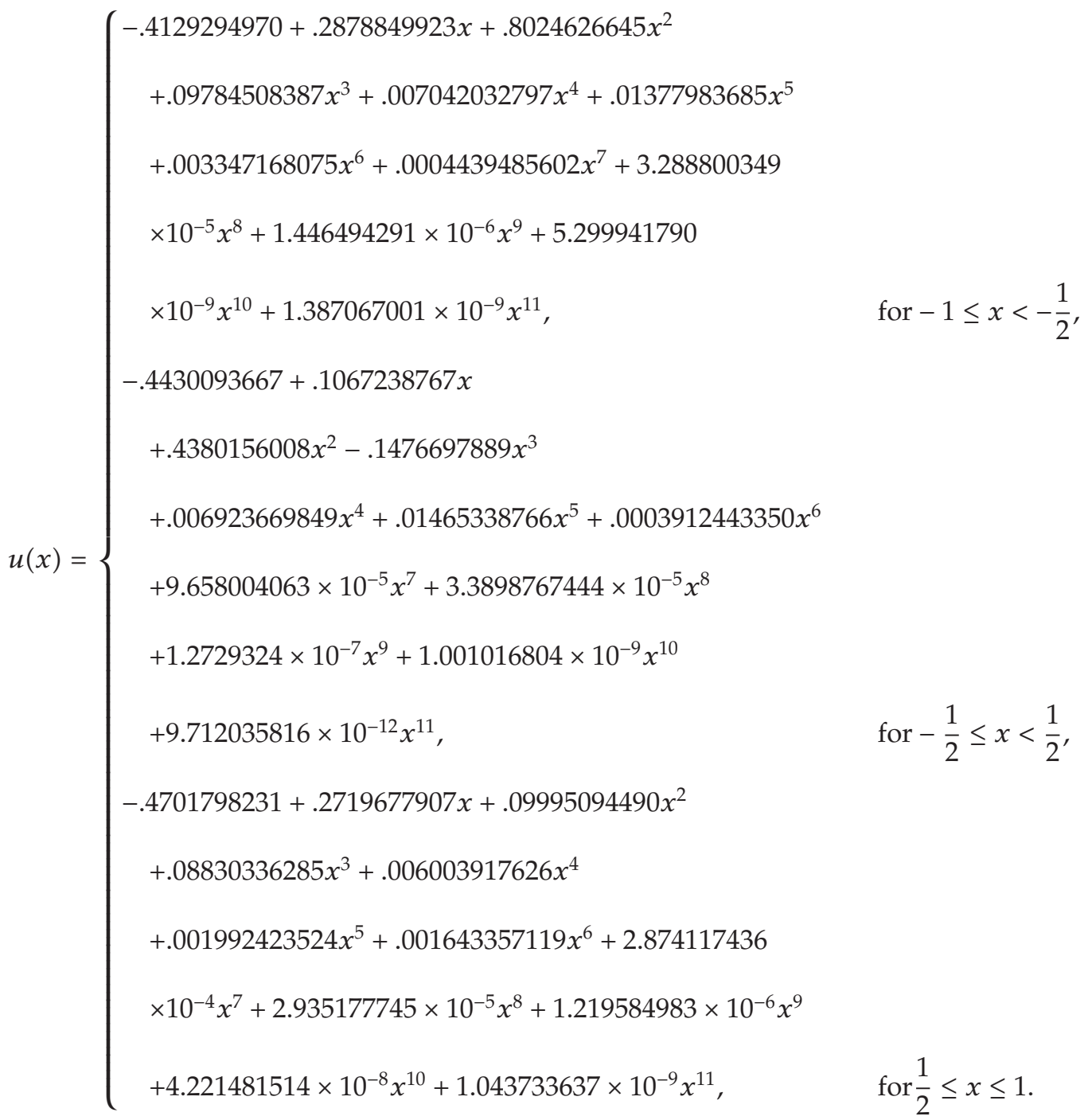




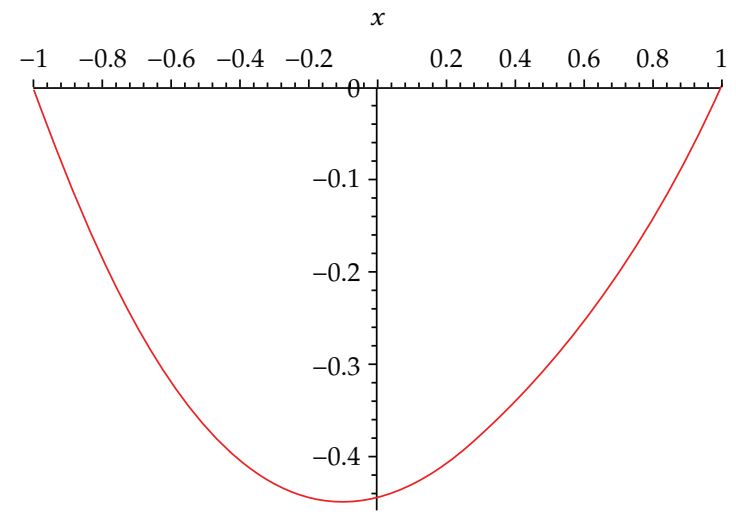

Figure 1

Figure 1 a graphical representation of analytical solution of system of third-order nonlinear boundary value problem (3.1) by using modified variation of parameters method.

Example 3.2. Consider following system of third-order nonlinear boundary value problem relevant to system (1.2):

$$
u^{\prime \prime \prime}= \begin{cases}-1+u+2 u^{3}, & \text { fo }-1 \leq x<-\frac{1}{2} \\ 2 u^{3}, & \text { for }-\frac{1}{2} \leq x<\frac{1}{2} \\ -1+u+2 u^{3}, & \text { for } \frac{1}{2} \leq x \leq 1,\end{cases}
$$

with boundary conditions $u(-1)=u(1)=0, u^{\prime}(-1)=1$.

Proceeding as before, we have a following iterative scheme to solve nonlinear system (3.13) by using the modified variation of parameters method:

$$
u_{k+1}(x)= \begin{cases}u_{k}(x)+\int_{0}^{x}\left(\frac{x^{2}}{2 !}-x s+\frac{s^{2}}{2 !}\right)\left(-1+u_{k}+A_{k}\right) d s, & \text { for }-1 \leq x<-\frac{1}{2} \\ u_{k}(x)+\int_{0}^{x}\left(\frac{x^{2}}{2 !}-x s+\frac{s^{2}}{2 !}\right)\left(A_{k}\right) d s, & \text { for }-\frac{1}{2} \leq x<\frac{1}{2} \\ u_{k}(x)+\int_{0}^{x}\left(\frac{x^{2}}{2 !}-x s+\frac{s^{2}}{2 !}\right)\left(-1+u_{k}+A_{k}\right) d s, & \text { for } \frac{1}{2} \leq x \leq 1 .\end{cases}
$$

Case $1(-1 \leq x<-1 / 2)$. In this case, we implement MVPM as follows. We consider the initial value as

$$
u_{0}=c_{2} x
$$


and obtain further iterations as follows:

$$
\begin{aligned}
u_{1}(x) & =c_{1} \frac{x^{2}}{2 !}+c_{3}+\int_{0}^{x}\left(\frac{x^{2}}{2 !}-x s+\frac{s^{2}}{2 !}\right)\left(A_{0}+u_{0}-1\right) d s \\
u_{1}(x) & =c_{3}+\frac{1}{2} c_{1} x^{2}-\frac{1}{6} x^{3}+\frac{1}{24} c_{2} x^{4}+\frac{1}{60} c_{2}{ }^{3} x^{6} \\
u_{k+2}(x) & =\int_{0}^{x}\left(\frac{x^{2}}{2 !}-x s+\frac{s^{2}}{2 !}\right)\left(A_{k+1}+u_{k+1}\right) d s, \quad \text { for } k=0,1,2, \ldots, \\
u_{2}(x) & =\frac{1}{6} c_{3} x^{3}+\left(\frac{1}{120} c_{1}+\frac{1}{10} c_{2}{ }^{2} c_{3}\right) x^{5}-\frac{1}{720} x^{6}+\left(\frac{1}{70} c_{2} c_{1}+\frac{1}{5040}\right) c_{2} x^{7} \\
& -\frac{1}{336} c_{2}{ }^{2} x^{8}+\frac{1}{1890} c_{2}{ }^{3} x^{9}+\frac{1}{9900} c_{2}{ }^{5} x^{11},
\end{aligned}
$$

Case $2(-1 / 2 \leq x<1 / 2)$. In this case, we have following approximations:

$$
\begin{aligned}
u_{0} & =c_{5} x \\
u_{1}(x) & =c_{4} \frac{x^{2}}{2 !}+c_{6}+\int_{0}^{x}\left(\frac{x^{2}}{2 !}-x s+\frac{s^{2}}{2 !}\right)\left(A_{0}\right) d s \\
u_{1}(x) & =c_{6}+\frac{1}{2} c_{4} x^{2}+\frac{1}{60} c_{5}^{3} x^{6} \\
u_{k+2}(x) & =\int_{0}^{x}\left(\frac{x^{2}}{2 !}-x s+\frac{s^{2}}{2 !}\right)\left(A_{k+1}\right) d s, \text { for } k=0,1,2, \ldots, \\
u_{2}(x) & =\frac{1}{10} c_{5}{ }^{2} c_{6} x^{5}+\frac{1}{70} c_{5}{ }^{2} c_{4} x^{7}+\frac{1}{9900} c_{5}{ }^{5} x^{11},
\end{aligned}
$$

Case $3(1 / 2 \leq x \leq 1)$. In this case, we proceed as follows:

$$
\begin{aligned}
u_{0} & =c_{8} x \\
u_{1}(x) & =c_{7} \frac{x^{2}}{2 !}+c_{9}+\int_{0}^{x}\left(\frac{x^{2}}{2 !}-x s+\frac{s^{2}}{2 !}\right)\left(A_{0}+u_{0}-1\right) d s \\
u_{1}(x) & =c_{9}+\frac{1}{2} c_{7} x^{2}-\frac{1}{6} x^{3}+\frac{1}{24} c_{8} x^{4}+\frac{1}{60} c_{8}{ }^{3} x^{6} \\
u_{k+2}(x) & =\int_{0}^{x}\left(\frac{x^{2}}{2 !}-x s+\frac{s^{2}}{2 !}\right)\left(A_{k+1}+u_{k+1}\right) d s, \text { for } k=0,1,2, \ldots, \\
u_{2}(x) & =\frac{1}{6} c_{9} x^{3}+\left(\frac{1}{120} c_{7}+\frac{1}{10} c_{8}{ }^{2} c_{9}\right) x^{5}-\frac{1}{720} x^{6}+\left(\frac{1}{70} c_{8} c_{7}+\frac{1}{5040}\right) c_{8} x^{7} \\
& -\frac{1}{336} c_{8}{ }^{2} x^{8}+\frac{1}{1890} c_{8}{ }^{3} x^{9}+\frac{1}{9900} c_{8}{ }^{5} x^{11}
\end{aligned}
$$


Hence, we have the following series solution after two iterations

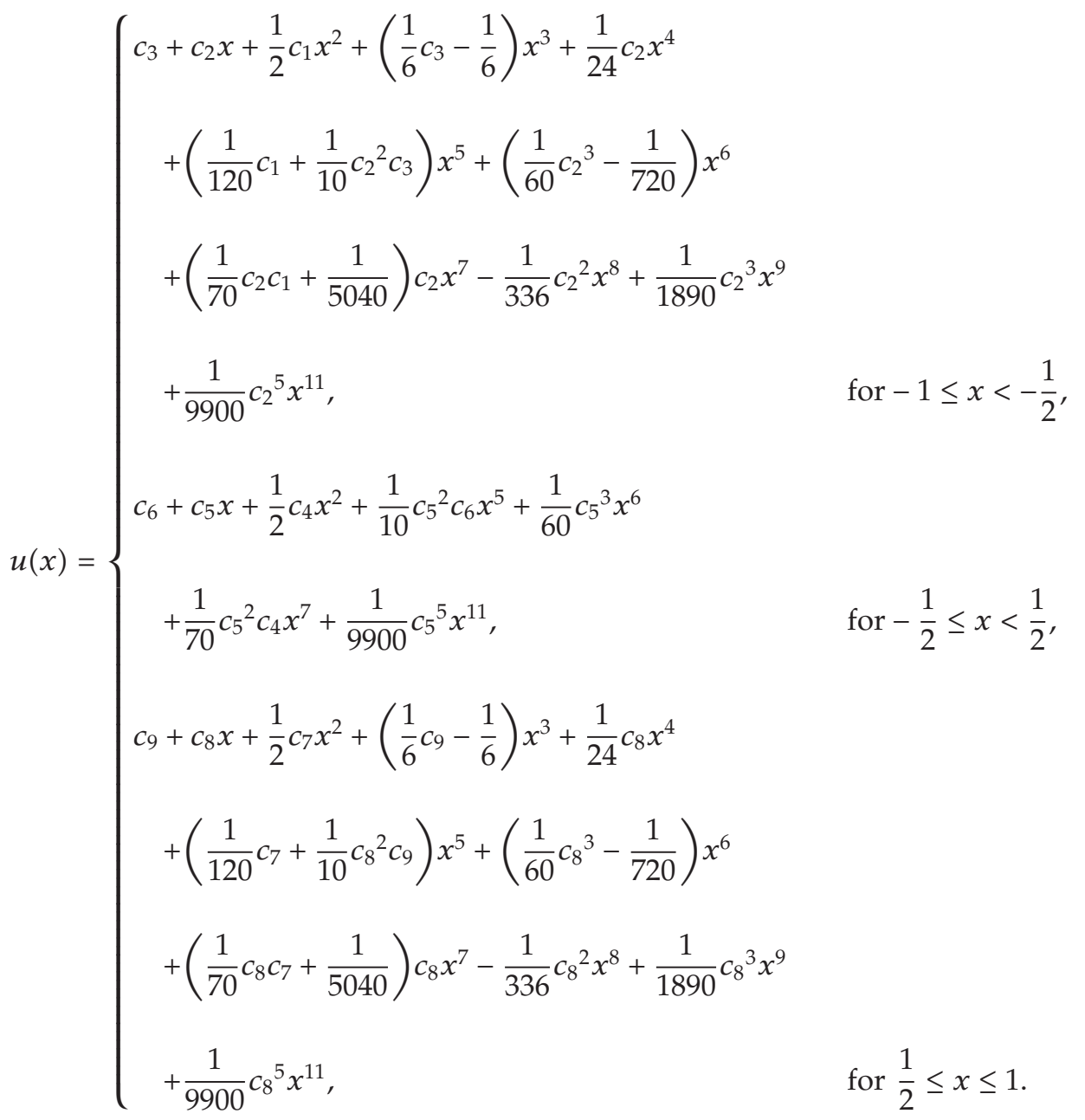

By using boundary conditions and continuity conditions at $x=-1 / 2$ and $x=1 / 2$ and we have a system of nonlinear equations. By using Newton's method for system of nonlinear equations, we have the following values of unknown constants:

$$
\begin{array}{lll}
c_{1}=-1.3277592790, & c_{2}=-.0523372235, & c_{3}=.5249671414, \\
c_{4}=-1.0719942050, & c_{5}=.0140953604, & c_{6}=.5362933242, \\
c_{7}=-.8198949242, & c_{8}=-.0519640842, & c_{9}=.5476022141 .
\end{array}
$$

By using values of unknowns from (3.20) into (3.19), we have following analytic solution of system of third-order nonlinear boundary value problem associated with obstacle 


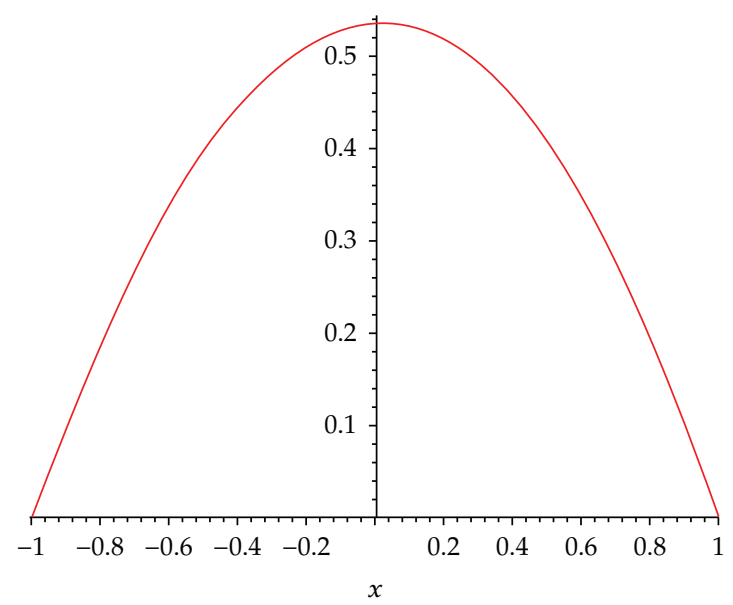

Figure 2

problem (3.13)

$$
u(x)=\left\{\begin{array}{l}
.5249671414-.0523372235 x-.6638796395 x^{2} \\
-.07917214313 x^{3}-.002180717646 x^{4}-.01092086245 x^{5} \\
\quad-.001391278245 x^{6}-6.234120193 \times 10^{-5} x^{7}-8.15233620 \\
\quad \times 10^{-6} x^{8}-7.585255857 \times 10^{-8} x^{9}-3.966598131 \times 10^{-11} x^{11}, \quad \text { for }-1 \leq x<-\frac{1}{2} \\
.5362933242+.0140953604 x-.5359971025 x^{2} \\
\quad+1.065503205 \times 10^{-5} x^{5}+4.667424523 \times 10^{-8} x^{6} \\
-3.042613354 \times 10^{-6} x^{7}+5.620121814 \times 10^{-14} x^{11}, \\
.5476022141-.0519640842 x-.4099474621 x^{2} \\
\quad-.07539963101 x^{3}-.002165170175 x^{4}-.006684590535 x^{5} \\
-.001391227503 x^{6}-4.193796883 \times 10^{-5} x^{7}-8.036506092 \\
\quad \times 10^{-5} x^{8}-7.424172074 \times 10^{-8} x^{9}-3.827200321 \times 10^{-11} x^{11}, \quad \text { for } \frac{1}{2} \leq x \leq 1 .
\end{array}\right.
$$

Figure 2 is a graphical representation of analytical solution of system of thirdorder nonlinear boundary value problem (3.13) by using modified variation of parameters method. 


\section{Conclusion}

In this paper, we have used the modified variation of parameters method, which is a combination of variation of parameters method and Adomian's decomposition method for solving system of third-order nonlinear boundary value problem. It is worth mentioning that we have solved nonlinear systems of boundary value problem by our proposed technique while most of the methods in the literature are proposed to solve linear systems of boundary value problems associated with obstacle problems. We took two examples for both the systems which are highly nonlinear in their nature. After applying our proposed technique we obtained series solutions as well as their graphical representation over the whole domain. We analyze that our proposed method is well suited for such physical problems as it provides best solution in less number of iterations. It is worth mentioning that the method is capable of reducing the volume of the computational work as compared to the existing classical methods. The use of multiplier gives this technique a clear edge over the decomposition method by removing successive application of integrals. Therefore, it may be concluded that modified variation of parameters method is very powerful and efficient technique for finding the analytical solutions for a wide class of systems of nonlinear boundary value problems. We would also like to mention that Ma et al. [26, 27] have used the multiple expo function method and linear superposition principle for solving the Hirota bilinear equations for constructing a specific subclass of $\mathrm{N}$-soliton solutions. It is an interesting and open problems to compare the modified variation of parameters method with the technique of Ma et al. [26, 27] for solving the system of third-order nonlinear boundary value problems associated with variational inequalities. Applications of the multi-expo function method for solving the variational inequalities and related optimization problems is an interesting problem for future research. Results proved in this paper may inspire the research for novel and innovative applications of these techniques.

\section{Acknowledgments}

The authors would like to thank the referee for his/her very useful and constructive comments. The authors are also grateful to Dr. S. M. Junaid Zaidi, Rector, CIIT for providing the excellent research facilities. This research is supported by the Visiting Professor Program of King Saud University, Riyadh, Saudi Arabia and Research Grant no. KSU.VPP. 108.

\section{References}

[1] F. Gao and C. M. Chi, "Solving third-order obstacle problems with quartic B-splines," Applied Mathematics and Computation, vol. 180, no. 1, pp. 270-274, 2006.

[2] M. A. Noor, "Some developments in general variational inequalities," Applied Mathematics and Computation, vol. 152, no. 1, pp. 199-277, 2004.

[3] M. A. Noor, Principles of Variational Inequalities, Lap-Lambert Academic, Saarbrucken, Germany, 2009.

[4] M. A. Noor, "Extended general variational inequalities," Applied Mathematics Letters, vol. 22, no. 2, pp. 182-185, 2009.

[5] M. A. Noor, K. I. Noor, A. Waheed, and E. Al-Said, "Modified variation of parameters method for solving a system of second-order nonlinear boundary value problems," International Journal of Physical Sciences, vol. 5, no. 16, pp. 2426-2431, 2010.

[6] M. A. Noor, K. I. Noor, A. Waheed, and E. Al-Said, "On computation methods for solving systems of fourth-order nonlinear boundary value problems," International Journal of Physical Sciences, vol. 6, no. 1, pp. 128-135, 2011.

[7] M. A. Noor, K. I. Noor, and T. M. Rassias, "Some aspects of variational inequalities," Journal of Computational and Applied Mathematics, vol. 47, no. 3, pp. 285-312, 1993. 
[8] M. A. Noor and A. K. Khalifa, "A numerical approach for odd-order obstacle problems," International Journal of Computer Mathematics, vol. 54, pp. 109-116, 1994.

[9] M. A. Noor and E. A. Al-Said, "Numerical solutions of fourth order variational inequalities," International Journal of Computer Mathematics, vol. 75, no. 1, pp. 107-116, 2000.

[10] M. A. Noor, S. T. Mohyud-Din, and A. Waheed, "Variation of parameters method for solving fifthorder boundary value problems," Applied Mathematics and Information Sciences, vol. 2, no. 2, pp. 135$141,2008$.

[11] S. Islam, M. A. Khan, I. A. Tirmizi, and E. H. Twizell, “Non polynomial spline approach to the solution of a system of third-order boundary-value problems," Applied Mathematics and Computation, vol. 168, no. 1, pp. 152-163, 2005.

[12] E. A. Al-Said and M. A. Noor, "Computational methods for fourth order obstacle boundary value problems," Communications on Applied Nonlinear Analysis, vol. 2, no. 3, pp. 73-83, 1995.

[13] E. A. Al-Said and M. A. Noor, "Quartic spline method for solving fourth order obstacle boundary value problems," Journal of Computational and Applied Mathematics, vol. 143, no. 1, pp. 107-116, 2002.

[14] E. A. Al-Said, M. A. Noor, D. Kaya, and K. Al-Khaled, "Finite difference method for solving fourthorder obstacle problems," International Journal of Computer Mathematics, vol. 81, no. 6, pp. 741-748, 2004.

[15] A. K. Khalifa and M. A. Noor, "Quintic splines solutions of a class of contact problems," Mathematical and Computer Modelling, vol. 13, no. 2, pp. 51-58, 1990.

[16] S. T. Mohyud-Din, M. A. Noor, and A. Waheed, "Variation of parameters method for solving sixthorder boundary value problems," Communications of the Korean Mathematical Society, vol. 24, no. 4, pp. 605-615, 2009.

[17] S. T. Mohyud-Din, M. A. Noor, K. I. Noor, and A. Waheed, "Modified variation of parameters method for solving nonlinear boundary value problems," International Journal of Modern Physics B, 2011.

[18] W. X. Ma and Y. You, "Solving the Korteweg-de Vries equation by its bilinear form: Wronskian solutions," Transactions of the American Mathematical Society, vol. 357, no. 5, pp. 1753-1778, 2005.

[19] W. X. Ma and Y. You, "Rational solutions of the Toda lattice equation in casoratian form," Chaos, Solitons and Fractals, vol. 22, no. 2, pp. 395-406, 2004.

[20] W. X. Ma, C. X. Li, and J. S. He, "A second Wronskian formulation of the Boussinesq equation," Nonlinear Analysis, vol. 70, no. 12, pp. 4245-4258, 2009.

[21] J. I. Ramos, "On the variational iteration method and other iterative techniques for nonlinear differential equations," Applied Mathematics and Computation, vol. 199, no. 1, pp. 39-69, 2008.

[22] T. A. Abassy, M. A. El-Tawil, and H. El Zoheiry, "Toward a modified variational iteration method," Journal of Computational and Applied Mathematics, vol. 207, no. 1, pp. 137-147, 2007.

[23] A. M. Wazwaz, "Approximate solutions to boundary value problems of higher order by the modified decomposition method," Computers and Mathematics with Applications, vol. 40, no. 6-7, pp. 679-691, 2000.

[24] M. A. Noor, "General variational inequalities," Applied Mathematics Letters, vol. 1, no. 2, pp. 119-121, 1988.

[25] M. A. Noor, "Variational inequalities in physical oceanography," in Ocean Wave Engineering, M. Rahman, Ed., pp. 201-226, Computational Mechanics, Southampton, UK, 1994.

[26] W. X. Ma and E. Fan, "Linear superposition principle applying to Hirota bilinear equations," Computers and Mathematics with Applications, vol. 61, pp. 950-959, 2011.

[27] W. X. Ma, T. Huang, and Y. Zhang, "A multiple exp-function method for nonlinear differential equations and its applications," Physica Scripta, vol. 82, no. 6, article 065003, 2010. 


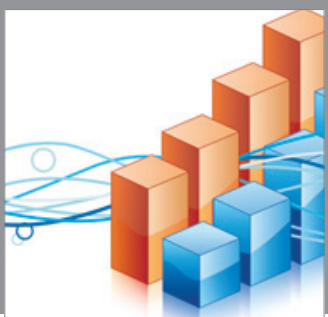

Advances in

Operations Research

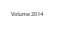

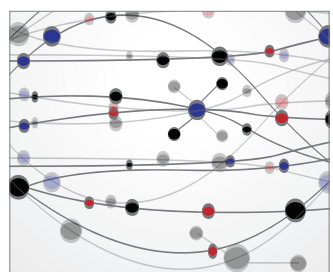

\section{The Scientific} World Journal
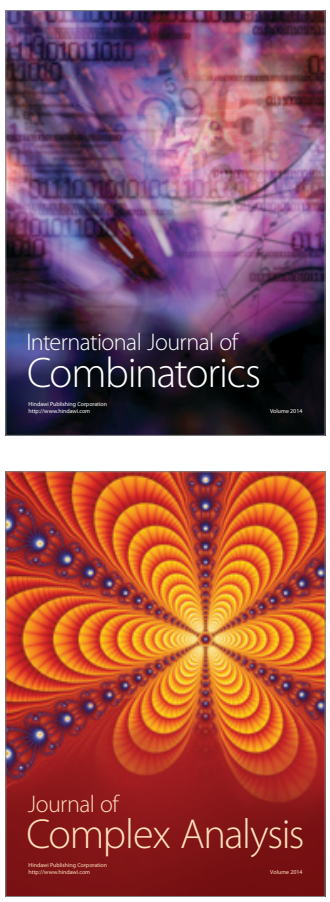

International Journal of

Mathematics and

Mathematical

Sciences
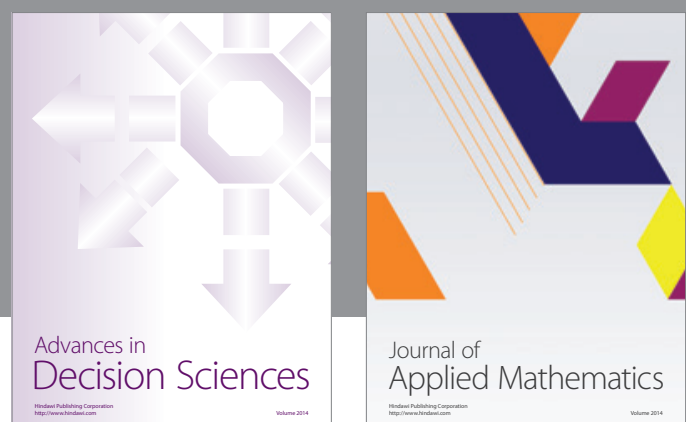

Journal of

Applied Mathematics
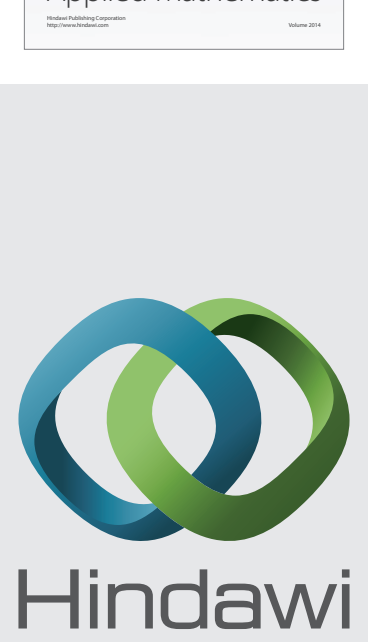

Submit your manuscripts at http://www.hindawi.com
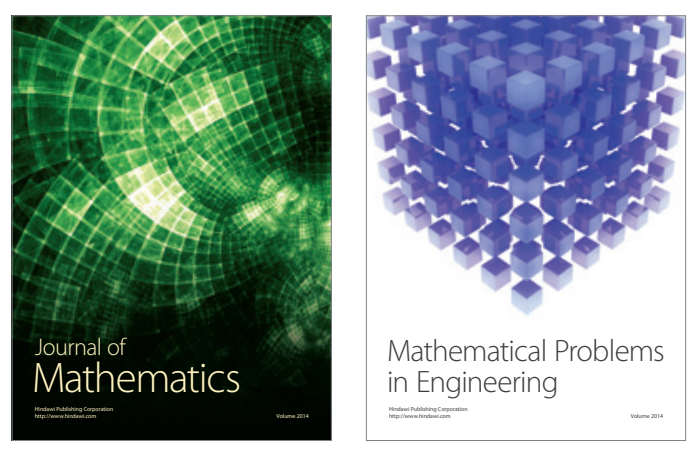

Mathematical Problems in Engineering
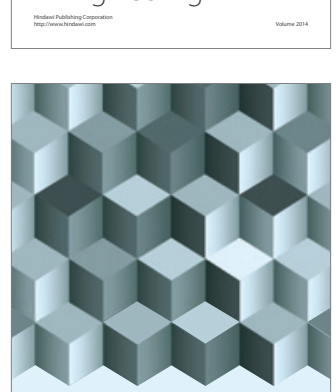

Journal of

Function Spaces
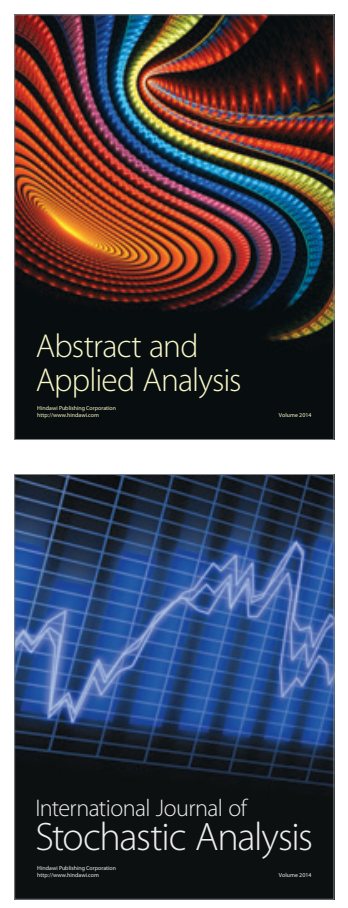

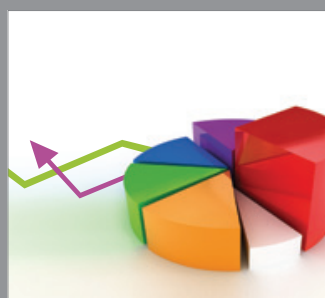

ournal of

Probability and Statistics

Promensencen
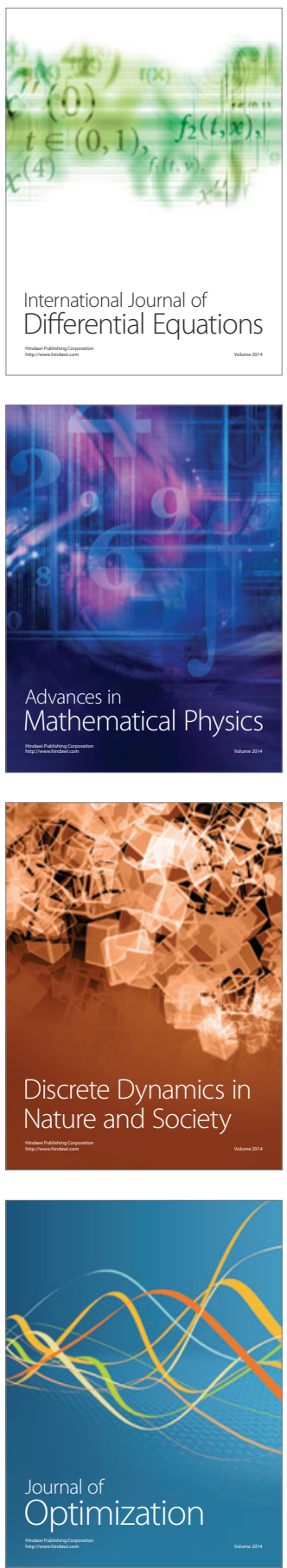